\title{
Cardiac strangulation following epicardial pacemaker implantation: A rare pediatric complication
}

\author{
Erick M. Carreras, ${ }^{\mathrm{a}, \mathrm{b}}$ Walter J. Duncan, MD, FRCP(C), FACC, ${ }^{\mathrm{c}, \mathrm{d}}$ Ognjenka Djurdjev, BAH, MSc, ${ }^{\mathrm{e}}$ and \\ Andrew I. M. Campbell, BSc, MD, $\operatorname{FRCS}(C)^{\mathrm{a}, \mathrm{b}}$
}

\begin{abstract}
Objectives: The aim of our study was 2-fold: to determine the incidence of cardiac strangulation (CS) and to develop a clinical pathway to aid in the diagnosis and prognosis of CS. In $<2$ years, 2 cases of CS occurred in our institution, which caused much alarm and led to the study's objectives.
\end{abstract}

\begin{abstract}
Methods: All patients who underwent implantation of an epicardial pacemaker from January 1992 to March 2012 were included. There were no exclusion criteria. Health records were used to locate all subjects and gather all retrospective data. Prospectively, subjects without a chest radiograph from the previous 2 years were approached for imaging.
\end{abstract}

Results: This study included 86 patients retrospectively, and 84 patients prospectively. There was a $2.3 \%$ incidence, and a $1.2 \%$ mortality, related to CS. A pattern of posterior looping of the ventricular lead was seen in radiographs of both CS-diagnosed patients. Five variables were significantly associated with an outcome of CS $(P=.0153)$.

Conclusions: Our data indicate that the 2 cases of CS were not caused by a lack of follow-up but by a lack of consistent imaging for diagnosis. This conclusion is supported by the 8 cases of CS found in the Englishlanguage literature. If the patient is age $\leq 6$ months at the time of implantation, particular attention should be given to the placement of leads and follow-up. (J Thorac Cardiovasc Surg 2015;149:522-7)

See related commentary on pages 528-9.

Cardiac strangulation (CS) is a mechanical complication that can occur with epicardial pacemaker (EP) systems in any individual undergoing somatic growth, by causing the entrapment of the patient's heart or great vessels. CS occurs when the epicardial leads of a pacemaker adhere to the heart in a circumferential manner and over time constrict the encompassed structures. As the patient grows, the lead tightens around the heart, and depending on the location of maximal compression, this may lead to coronary

From the Division of Pediatric Cardiovascular and Thoracic Surgery, ${ }^{a}$ Department of Surgery, BC Children's Hospital, Provincial Health Services Authority, Vancouver, British Columbia, Canada; Division of Cardiovascular Surgery, ${ }^{\mathrm{b}}$ Department of Surgery, Faculty of Medicine, University of British Columbia, Vancouver, British Columbia, Canada; Division of Cardiology, ${ }^{c}$ Department of Pediatrics, BC Children's Hospital, Provincial Health Services Authority, Vancouver, British Columbia, Canada; Division of Cardiology, ${ }^{\mathrm{d}}$ Department of Pediatrics, Faculty of Medicine, University of British Columbia, Vancouver, British Columbia, Canada; and Department of Measurement \& Reporting, ${ }^{\text {e }}$ Provincial Health Service Authority, Vancouver, British Columbia, Canada.

This work was supported by a grant from Innovations in Acute Care and Technology (iACT), Vancouver, British Columbia, Canada.

Disclosures: Authors have nothing to disclose with regard to commercial support.

Received for publication May 15, 2014; revisions received Oct 17, 2014; accepted for publication Oct 20, 2014; available ahead of print Dec 10, 2014.

Address for reprints: Andrew I. M. Campbell, BSc, MD, FRCS(C), BC Children's

Hospital, Provincial Health Services Authority, AB306-4480 Oak St, Vancouver,

British Columbia, Canada V6H 3V4 (E-mail: acampbell@cw.bc.ca).

0022-5223/\$36.00

Copyright (c) 2015 by The American Association for Thoracic Surgery

http://dx.doi.org/10.1016/j.jtcvs.2014.10.094 stenosis, valvular insufficiency, or ventricular dysfunction, with the potential of cardiac arrest and death. ${ }^{1-8}$ As somatic growth is necessary for CS to occur, this mechanical complication is limited to the pediatric population.

Globally, CS is considered a rare pediatric complication, with only 8 published cases. ${ }^{1-8}$ However, there are 2 known cases at our institution, suggesting a much higher local incidence than the number of cases currently reported in the literature. Thus, the primary objective of the current study was to investigate the true incidence of CS, with the hypothesis that, based on the local incidence of CS, this complication has simply been underreported on a global scale as a result of its rarity on an individual institutional basis. The secondary objective was to develop a clinical pathway to aid in the diagnosis and follow-up of patients at risk of CS. As chest radiographs are noninvasive and have a minimal number of associated complications, yet provide some of the most explicit images of the epicardial leads, a standard posteroanterior (PA) and lateral chest radiograph was presumed to be the most practical method of identifying early signs of CS. ${ }^{9}$

\section{METHODS}

This study goes back 20 years, for 2 reasons: to cover at least one cohort of patients transitioning from pediatric to adult cardiac care; and because patients are not known to be clear of risk of CS at any specific age. The subject inclusion criteria were being a patient who had an epicardial pacemaker (EP) implantation, from January 1, 1992, to May 1, 2012, at BC Children's Hospital. Health records at this hospital were searched to 


\section{Abbreviations and Acronyms \\ $\mathrm{CI}=$ confidence interval \\ $\mathrm{CS}=$ cardiac strangulation \\ $\mathrm{CT}=$ computed tomography \\ $\mathrm{EP}=$ epicardial pacemaker \\ $\mathrm{PA}=$ posteroanterior \\ $\mathrm{SD}=$ standard deviation}

locate all patients who met these criteria. Medical records from all possible sources were reviewed to obtain all retrospective data on these patients, including cardiac surgical charts, pediatric cardiology charts, pacemaker interrogation charts, radiological records, and transfer charts. Transfer charts are for those who have been transferred to the Pacific Adult Congenital Heart clinic in St Paul's Hospital. This search process identified 86 patients.

This investigation included both retrospective and prospective data collection. In the retrospective portion, the patients' health records were abstracted for demographics, surgical details, complications, and all available follow-up data. The prospective portion involved reviewing a standard set of PA and lateral chest radiographs for all patients, but only if such a set or the fluoroscopic equivalent had not been obtained within the past 2 years. This process left 48 subjects without a recent chest radiograph or fluoroscopic equivalent. These subjects were invited to participate in the study and provide informed consent for imaging to be obtained. The chest radiographs were then reviewed to determine if potential CS was present or suspected. If so, a low-dose, contrast computed tomography (CT) scan was recommended to the patient's cardiologist or corresponding doctor. In all cases, subjects had imaging results explained to them. The Research Ethics Board of the University of British Columbia for the Children's \& Women's Health Centre of British Columbia approved this study (case number H12-01641).

\section{Statistics}

Exploratory data analysis was performed using descriptive measures. Categoric variables were expressed in terms of percentages with $95 \%$ confidence interval (CI). Continuous variables were expressed as medians with ranges because of their skewed distribution.

The incidence was reported with Clopper-Pearson exact CI. The strength of a statistical association was measured by use of the exact logistic regression with penalized likelihood method, by Firth. To ensure equal exposure, these analyses were restricted to cases that had $\geq 3$ years of follow-up, which left 66 subjects from the total subject pool of 86 . The 2 -sided $P$ value of $<.05$ was considered statistically significant. All analyses were performed using SAS software, version 9.3 (SAS Institute Inc, Cary, NC).

\section{RESULTS}

A classic pattern for an epicardial lead was recognized to exist in all CS-positive patients, both in the literature and in the local cases being presented. The pattern is denoted by posterior looping of the atrial and ventricular leads, forming a "heart-shaped" appearance on PA chest radiography (Figure 1, A).

\section{Literature Review}

The English-language literature was reviewed for reported cases of CS and published incidence. Key characteristics of reported cases were summarized (Table 1). A total of 100,900 epicardial leads from one manufacturer have been implanted in the United States between October 1981 and August 6, 2012 (Katie Lasch, Medtronic, personal communication, 2013). Assuming all implantations were of the most common type-a 2-lead implantation-and knowing that only 8 cases have been reported in the literature, the published global incidence was estimated to be $0.016 \%$. Two of the 86 identified patients had CS, resulting in a local incidence of $2.33 \%$ $(95 \%$ CI, $0.64 \%-8.09 \%)$, which is statistically significantly larger than $0.01 \%(P<.001)$. As 1 of the 2 cases is deceased, the resulting CS mortality rate was $1.16 \%(95 \%$ CI, $0.21 \%-6.30 \%)$.

\section{Case Details}

Within the study period, there were 2 cases of CS, and 1 case that required further investigation beyond the standard PA and lateral chest radiographs to eliminate CS as a potential risk. Patient 1 presented 3 years after implantation of an EP system for congenital heart block with new-onset

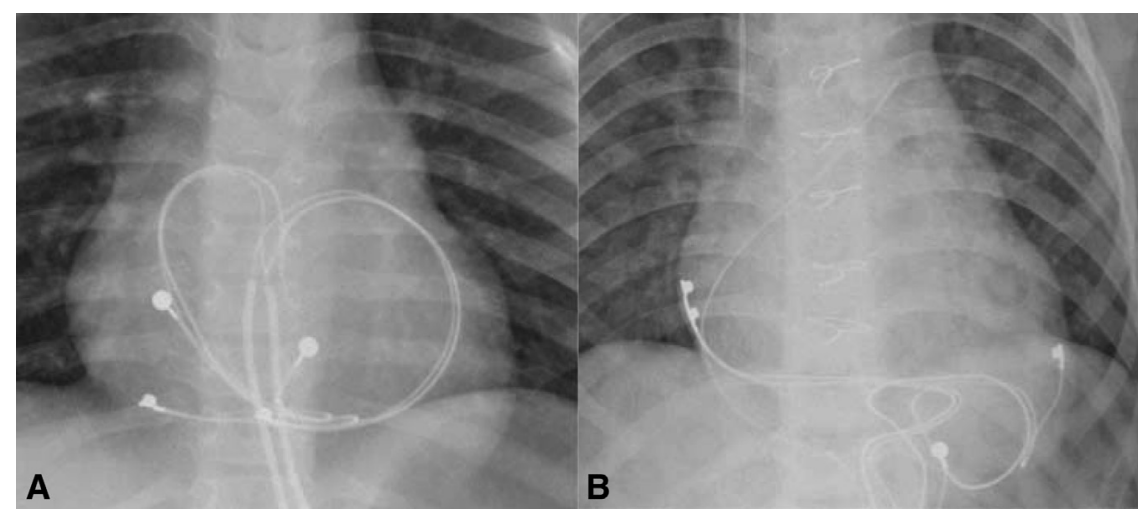

FIGURE 1. A, The last posteroanterior chest radiograph of patient 1 , confirming the diagnosis of CS, showing a classic pattern of posterior looping by the atrial and ventricular leads. B, Posteroanterior chest radiograph post-lead replacement, showing patient 1 clear of any possible CS with the leads well situated at the bottom of the pericardium. 
TABLE 1. Summary of literature review 1 of 2: Age at implantation of epicardial pacemaker, age at incidence of cardiac strangulation, and description of epicardial lead looping observed in each of the case studies identified during the literature review

\begin{tabular}{|c|c|c|c|}
\hline Case \# & Age at EP implantation & Age at CS & Epicardial lead looping notes \\
\hline 1 & $2 \mathrm{~d}$ & $3 \mathrm{y}$ & A posterior looping lead was compressing the anterior descending coronary artery. \\
\hline 2 & $8 \mathrm{mo}$ & $6 \mathrm{y}$ & An anterior looping lead was determined to have caused CS in this patient. \\
\hline 3 & "... as a neonate ..." & $9 \mathrm{y}$ & $\begin{array}{l}\text { The lead entered the pericardium posteriorly and crossed anteriorly over both the } \\
\text { circumflex and the anterior descending coronary arteries before inserting into the } \\
\text { right ventricle. There was also a fibrous sheath adherent to the myocardium creating } 2 \\
\text { points of attachment on either side of the coronary artery. }\end{array}$ \\
\hline 4 & $\begin{array}{l}\text { Approximately } 7 \mathrm{~d} \text { (“... at } 1 \\
\text { wk of life.") }\end{array}$ & $12 \mathrm{y}$ & $\begin{array}{l}\text { The lead completely encircled the cardiac silhouette on chest radiography. } \\
\text { Compression of the left anterior descending coronary artery confirmed by cardiac } \\
\text { catheterization. }\end{array}$ \\
\hline 5 & $6 \mathrm{~d}$ & $20 \mathrm{mo}$ & $\begin{array}{l}\text { The patient presented at } 20 \text { mo post-EP implantation with a 3-d history of intermittent } \\
\text { pain in the right arm, frequent falling, and syncope. Physical examination was } \\
\text { unremarkable, but chest radiography suggested a case of CS. The diagnosis was } \\
\text { confirmed at emergency surgery with the visualization of a deep trough made by the } \\
\text { EP lead in the atrioventricular groove. The patient was discharged } 2 \text { wk postrevision } \\
\text { without complications. }\end{array}$ \\
\hline 6 & $\begin{array}{l}\text { Within the first } 2 \mathrm{~d} \text { of life after } \\
\text { a } 37 \text {-wk gestation period-no } \\
\text { exact age provided }\end{array}$ & $10 \mathrm{mo}$ & $\begin{array}{l}\text { The patient was admitted for edema at the age of } 10 \mathrm{y} \text {. Several imaging techniques were } \\
\text { performed and suggested compression of the left anterior descending artery. } \\
\text { Selective left coronary angiography was performed, which resulted in ventricular } \\
\text { fibrillation. An emergency operation confirmed the EP lead encircling the posterior } \\
\text { atrioventricular groove, and demonstrated the lead was compressing the left } \\
\text { circumflex artery. The patient did not recover and expired on postoperative day } 6 \text {. }\end{array}$ \\
\hline 7 & $3 \mathrm{mo}$ & $2.67 \mathrm{y}$ & $\begin{array}{l}\text { The patient presented for evaluation for cardiac resynchronization therapy at the age of } \\
2.67 \mathrm{y} \text {. Selective angiography of the left coronary artery revealed CS caused by the } \\
\text { right ventricular electrode. It was determined there was systolic compression of a } \\
\text { diagonal branch, the circumflex artery, and posterolateral branch, as well as complete } \\
\text { occlusion of the distal left anterior descending artery. }\end{array}$ \\
\hline 8 & $2 \mathrm{mo}$ & $5 \mathrm{y}$ & $\begin{array}{l}\text { At the age of } 5 \text { during cardiac follow-up, the patient was noted to have a loud systolic } \\
\text { murmur. A chest radiograph revealed anterior looping of the EP lead and further } \\
\text { imaging indicated it was likely there was extracardiac compression at the level of the } \\
\text { pulmonary valve. Surgery performed to remove the EP system confirmed the } \\
\text { diagnosis of a lead compressing the pulmonary artery. }\end{array}$ \\
\hline
\end{tabular}

The case number corresponds to the number of the published reference regarding that case. For example, Case \#2 in the table corresponds to Reference 2 in the reference list. $E P$, Epicardial pacemaker; $C S$, cardiac strangulation.

tricuspid regurgitation and extrinsic right ventricular compression on echo. These symptoms prompted a re-examination of her chest radiographs that revealed a classic pattern of posterior looping of the atrial and ventricular leads (Figure 1, A). This patient underwent urgent cardiac surgery via a median sternotomy to remove and replace the old leads; this procedure was completed without incident (Figure 1, B). It was 3 years between the most recent CS-negative radiograph for patient 1 , and the radiograph that resulted in a CS-positive diagnosis for patient 1 .

Patient 2 presented with a terminal case of CS. Patient 2 was 7 years post-EP implantation for congenital heart block, and had good exercise tolerance, with no complaints of chest pain. After aggressive physical exertion, the patient complained of chest pain and collapsed. Cardiopulmonary resuscitation was initiated almost immediately. After an extended effort at resuscitation, the patient was pronounced dead and a postmortem was obtained. The typical posterior looping into a "heart-shape" by the ventricular leads can be seen from the last antemortem chest radiograph (Figure 2, A). The images from the postmortem pathology report clearly show the extrinsic compression of the circumflex coronary artery by the ventricular lead (Figure 2, B), and the same view after removal of all epicardial leads and a barium injection (Figure 2, C). Cardiac strangulation by epicardial pacing leads was determined to be the cause of death by the pathologist and the coroner. It was 3 years between the most recent CS-negative radiograph for patient 2 , and the radiograph that resulted in a CS-positive diagnosis for patient 2.

Patient 3 had undergone implantation of an epicardial system for very lengthy sinus pauses at age 18 months. Now, at 8 years postimplantation, patient 3 was assessed as one of the prospective subjects of this study who had 


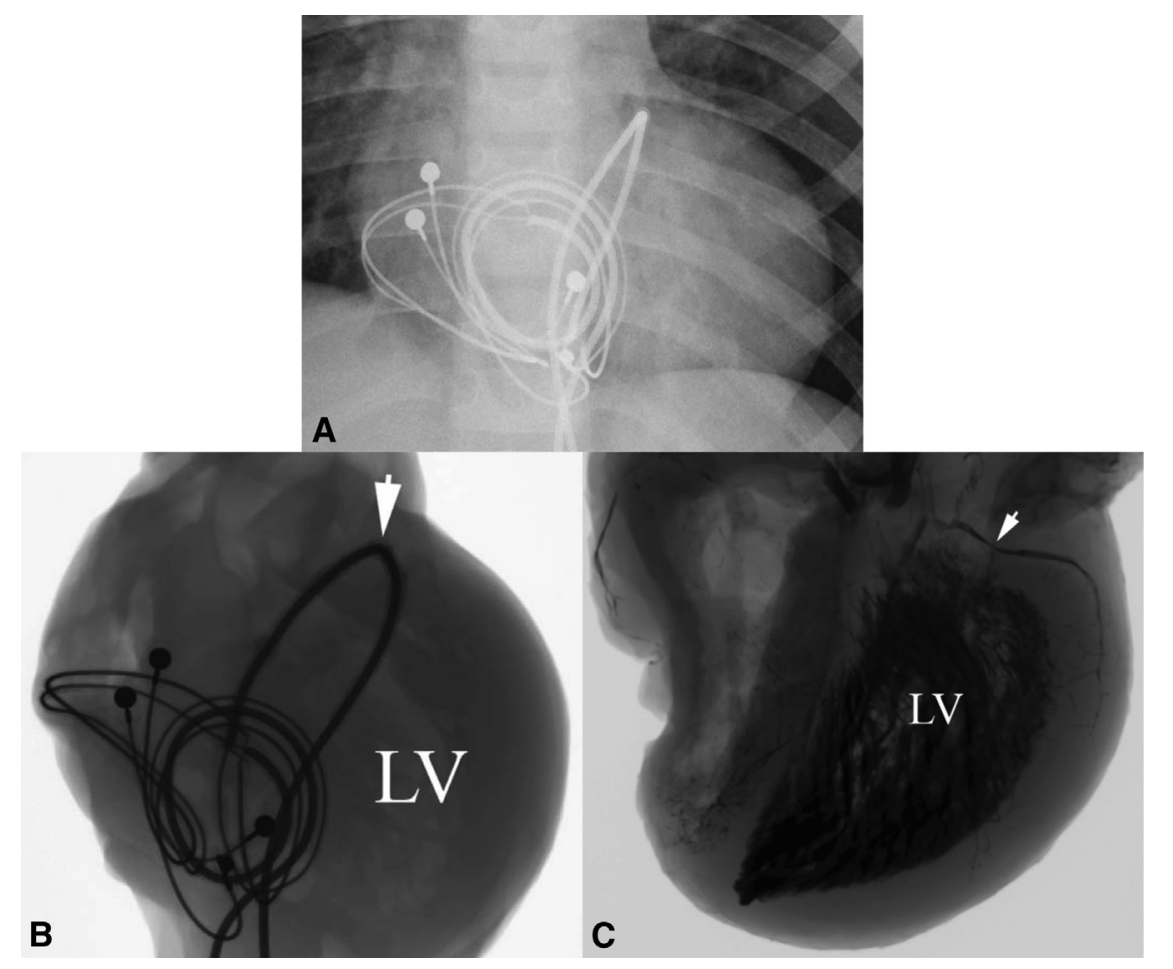

FIGURE 2. A, Last antemortem posteroanterior chest radiograph of patient 2. B, Postmortem cardiac radiograph of patient 2. The solid arrow indicates the common biventricular epicardial lead situated on the atrioventricular groove. C, Postmortem cardiac angiography, with barium, of patient 2, depicting the compression of the circumflex coronary artery by the common biventricular EP lead. The solid arrow shows the area of compression in the circumflex coronary artery that was injected with barium after postmortem removal of the EP and all associated leads. $L V$, Left ventricle.

not received a standard chest radiograph or fluoroscopic examination within the last 2 years. After consent was obtained, patient 3 was identified as being potentially at high risk of CS; however, a definitive diagnosis could not be made from these images alone (Figure $3, A$ and $B$ ). Therefore, a low-dose, low-contrast CT exam was performed. It was concluded that patient 3 was not at risk of CS, based on the CT images showing that the leads were clear of any circumferential looping around the heart, and had sufficient space for somatic growth (Figure 3,C). The only chest radiograph from this patient after EP implantation is the one from the current study, a chest radiography interval of 7 years.

A total of 86 patients underwent EP implantations during the 20-year study period. The age at EP implantation ranged from 1 day to 19.9 years (median: 15 months). The indications for all EPs have been grouped into 3 broad categories: congenital heart block, postoperative heart block, and rhythm disturbance. The incidence for each of these indications, respectively, was found to be $17(20 \%)$, $41(48 \%)$, and $28(33 \%)$. Details of all other patient characteristics can be found in Table 2 .

\section{Complications}

Thirty-one complications were identified throughout the course of the postimplantation follow-up. The 4 most common complications were tachycardia $(13 ; 15 \%)$, postpericardiotomy syndrome $(8 ; 9 \%)$, revision of implant $(6 ; 7 \%)$, and wound infections $(6 ; 7 \%)$. A total of 9 mortalities occurred within our subject pool. Eight of these were secondary to complications other than CS, including renal failure, myocardial infarct, chronic lung disease, and transplant. Eight mortalities had CS eliminated as a possibility.

\section{Follow-up}

A review of the chest radiograph frequency, per patient, per follow-up appointment, showed that patients were undergoing chest radiography in only $16 \%$ of their follow-up appointments. Of the 86 subjects, 22 never underwent a chest radiograph at any point after EP lead implantation. Of the 22 subjects that had never undergone a chest radiograph postimplantation, 7 had died. We reviewed their cases during this study to rule out CS.

Of the remaining 64 subjects, 37 had undergone chest radiographs within the last 2 years; 27 had not. Of these 27 subjects, 1 had a fluoroscopic examination equivalent performed within the last 2 years; the other 26 were approached for consent to have a prospective chest radiograph at BC Children's Hospital. Of the 26 subjects, 2 chose not to participate, and 1 was lost to follow-up. The final subject's chest radiographs revealed a case of 


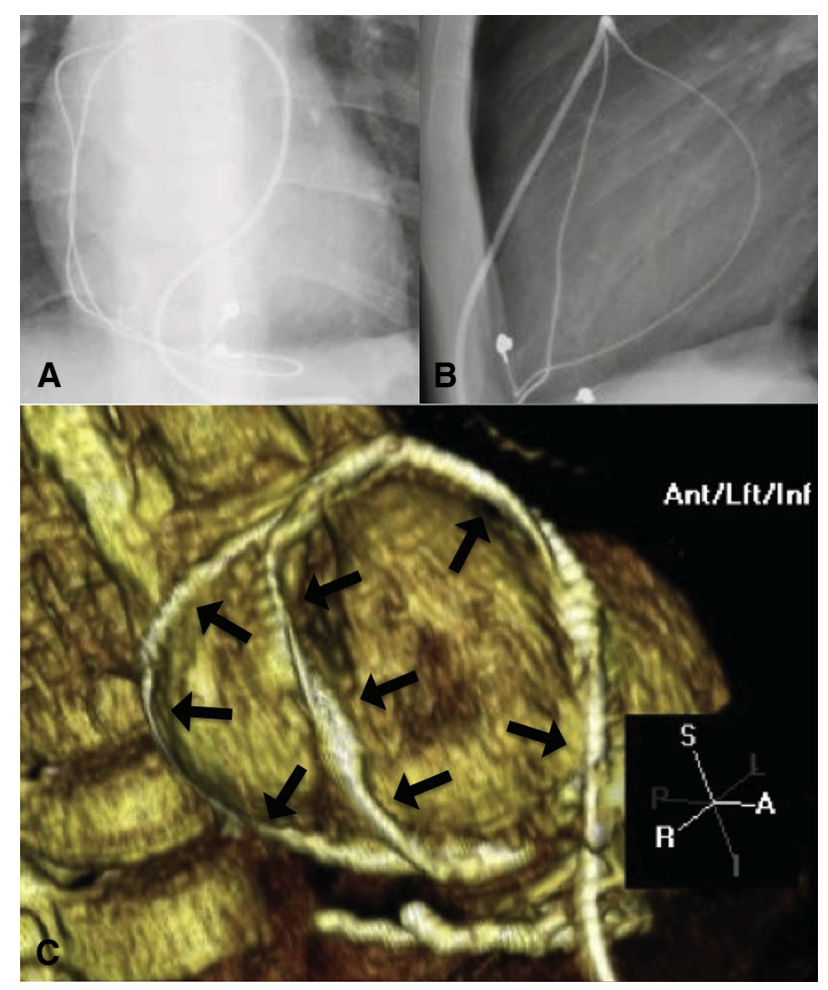

FIGURE 3. A, Posteroanterior chest radiograph of patient 3 that resulted from the prospective portion of the study protocol. B, The lateral chest radiograph of patient 3 that resulted from the prospective portion of the study protocol. C, Computed tomography of patient 3, revealing the anterior and lateral placement of the epicardial leads. Solid arrows indicate the location of the common and individual bipolar ventricular epicardial pacemaker leads. Ant, Anterior; $L f t$, left; Inf, inferior; $S$, superior; $L$, lateral; $P$, posterior; $A$, anterior; $R$, right; $I$, inferior.

query CS that led to the investigator's recommendation to the subject's cardiologist to prescribe a CT scan (Figure 3, C). Based on the CT imaging, patient 3 was considered not to be at risk of CS. After qualitative evaluation of the pacemaker interrogation reports, no

TABLE 2. Characteristics of patients who received epicardial implantations at BC Children's Hospital between January 1, 1992, and May 31, 2012

\begin{tabular}{lc}
\hline \multicolumn{1}{c}{ Variable } & $\mathbf{N}(\% ; \mathbf{9 5} \% \mathbf{C I})^{*}$ \\
\hline Age at EP implantation (mo) & Median: $15($ range: $0-239)$ \\
$<6$ & $26(30 ; 22-41)$ \\
$6-42$ & $30(35 ; 26-45)$ \\
$>42$ & $30(35 ; 26-45)$ \\
Female gender & $46(53 ; 42-64)$ \\
Weight $(\mathrm{kg})$ & Median: $8.5($ range: $1.7-59.7)$ \\
Weight $<7.3 \mathrm{~kg}(16.1 \mathrm{lb})$ & $37(43 ; 33-54)$ \\
Primary diagnosis & \\
Ventral septal defect & $13(15 ; 9-24)$ \\
Pulmonary atresia/stenosis & $10(12 ; 6-20)$ \\
Heart block, congenital included & $10(12 ; 6-20)$ \\
\hline$E P$, Epicardial pacemaker; $C I$, confidence interval. *Unless otherwise noted.
\end{tabular}

pacing parameters were identified that were consistent with CS, including changes in atrial and/or ventricular sensing thresholds, pacing thresholds, or lead impedance. A set of PA and lateral chest radiographs was a major contributing factor in confirming the need for further investigation.

Because of the small number of outcomes, we used exact logistic regression to assess the strength of association between potential risk factors and CS. None of the following tested parameters independently had a statistically significant association with the outcome of CS: age at EP implantation $<6$ months, weight at surgery $<7.3 \mathrm{~kg}$, the type of pacemaker implanted (a dual-chamber $\mathrm{EP})$, absence of postpericardiotomy syndrome, or absence of postoperative infections (Table 3). However, the presence of all 5 risk factors was associated with an approximately 17-fold increased risk of CS (median unbiased estimate of odds ratio $=16.88[95 \% \mathrm{CI}, 1.95$ to infinity], $P=.034$ ). Among 66 patients included in this analysis, 9 patients, including 2 confirmed cases of CS, had the combination of factors associated with a higher risk of CS: age $<6$ months at EP implantation, weight at surgery $<7.3 \mathrm{~kg}$, a dual-chamber pacemaker, no postpericardiotomy syndrome, and no postoperative infections.

\section{DISCUSSION}

Eight papers in the English-language literature outline cases of CS in North America and England (May 2012). It was estimated that the published CS incidence and mortality rate are $0.016 \%$ and $0.00396 \%$, respectively. In contrast, the resulting local CS incidence and mortality rate are $2.3 \%$ and $1.2 \%$, respectively. This difference in magnitude of both CS incidence and mortality rate resulted in the current study and a debate regarding the prevalence of this condition. It was recognized that CS is considered a rare mechanical complication in EPs, based on both the literature review and discussions with medical professionals across Canada and in the United States. We concluded that the likely cause of the lack of publication and initiative to raise awareness of this mechanical complication stems from the apparent lack of need for these, as CS may be overlooked as a cause of death in these patients. Hence, it is highly recommended that the possibility of CS not be disregarded during the follow-up care of EP patients until somatic growth has been completed.

The optimal method of EP implantation is to minimize the excess lead, if any, left within the pericardium, recognizing that with significant somatic growth, some small amount of redundancy is necessary. Any redundant length of lead that remains within the mediastinum should be placed on the anterior surface of the heart, and not along the diaphragm, where it may slip posterior to the apex 
TABLE 3. Factors associated with increased risk of cardiac strangulation

\begin{tabular}{lccr}
\hline \multicolumn{1}{c}{ Variable } & $\begin{array}{c}\text { Exact } \\
\text { odds } \\
\text { ratio }\end{array}$ & $\begin{array}{c}\mathbf{9 5} \% \\
\text { Confidence } \\
\text { interval }\end{array}$ & $\begin{array}{c}\boldsymbol{P} \\
\text { value }\end{array}$ \\
\hline Age at EP implantation $<6$ mo & 7.31 & $0.86-\infty$ & .13 \\
Weight at surgery $<7.3 \mathrm{~kg}$ & 3.83 & $0.45-\infty$ & .30 \\
Pacemaker type: dual-chamber & 0.92 & $0.11-\infty$ & 1.00 \\
Absence of postpericardiotomy & 0.38 & $0.04-\infty$ & 1.00 \\
$\quad$ syndrome & & & \\
Absence of postoperative infection & 6.58 & $0.00-59.43$ & 1.00 \\
\hline
\end{tabular}

Exact odds ratios are median unbiased estimates. EP, Epicardial pacemaker.

and potentially create a substrate for strangulation. The location of the battery is less significant, as long as it remains within a subcutaneous pocket where the majority of the excess pacemaker lead is contained.

Evaluation of a PA and lateral chest radiograph is an effective means to confirm or deny a diagnosis of CS. A crucial factor for successful implementation of this strategy is that chest radiography be performed consistently throughout the patient's somatic growth period-on average, until the patient has reached age 20 years. The rate of somatic growth is, of course, a function of the patient's age. But for practical considerations, we concluded that PA and lateral chest radiographs should be performed every 3 years during postimplantation follow-up if any redundant pacemaker lead is present in the pericardium.

This follow-up protocol is based on a qualitative observation of both epicardial lead displacement as a function of time, and the average somatic growth of a child. Regular chest radiographs are indicated as somatic growth progresses through the years from infancy to young adulthood. Particular attention should be given to those patients that have EP implantation before age 6 months, because of the large amount of somatic growth they will undergo, and the high likelihood of redundant pacemaker lead being left in the mediastinum.

\section{Limitations}

Procedural limitations included access to medical records, patients who moved or lived a great distance from our institution, and the small sample size of those in the outcome group who were positive for CS. Future research should include a multicenter study with a larger sample size, to improve statistical power and find single-variable predictors of CS.

\section{CONCLUSIONS}

The aim of our study was 2-fold: to determine the incidence of CS and to develop a clinical pathway to aid in the diagnosis and follow-up of patients at risk of this pathology. We have identified a significantly higher local incidence of CS compared with the published global estimates $(P<.001)$, and in our series, a local mortality of $1.16 \%$ was observed $(95 \%$ CI, $0.21 \%-6.30 \%)$. The clinical pathway determined to best aid the diagnosis of CS was a set of standard PA and lateral chest radiographs taken at a frequency of every 3 years. If CS is suspected from the resulting radiographs, a CT can be performed to confirm or refute the suspicion of CS.

The authors thank Angelica Oviedo and Jim Potts for their contributions.

\section{References}

1. Alhuzaimi A, Roy N, Duncan WJ. Cardiac strangulation from epicardial pacemaker: early recognition and prevention. Cardiol Young. 2011;4:471-3.

2. Eyeskens B, Mertens L, Moerman P, Ector H, Daenen W, Gewilling M. Cardiac strangulation, a rare complication of epicardial pacemaker leads during growth. Heart. 1997;3:288-9.

3. Salerno JC, Johnston TA, Chun TU, Jones TK. Coronary compression by an epicardial pacing lead within the pericardium. J Cardiovasc Electrophysiol. 2007;7:786.

4. Macicek SL, Cannon BC, Kyle WB, Krishnamurthy R, Breinholt JP, Ing FF. Dynamic coronary artery compression by pacemaker lead. Circulation. 2011;16:1792-4.

5. Brenner JI, Gaines S, Cordier J, Reiner BI, Haney PJ, Gundry SR. Cardiac strangulation: two-dimensional echo recognition of a rare complication of epicardial pacemaker therapy. Am J Cardiol. 1988;8:654-6.

6. Perry JC, Nihil MR, Ludomirsky A, Ott DA, Garson A Jr. The pulmonary artery lasso: epicardial pacing lead causing right ventricular outflow obstruction. Pacing Clin Electrophysiol. 1991;6:1018-23.

7. Watanabe H, Hayashi J, Sugawara M, Hashimoto T, Sato S, Takeuchi K. Cardiac strangulation in a neonatal case: a rare complication of permanent epicardial pacemaker leads. Thorac Cardiovasc Surg. 2000;2:103-5.

8. Reide FT, Kostelka M, Dähnert I. Cardiac strangulation: a rare, but devastating complication of epicardial pacing causing progressive myocardial ischemia. Eur Heart. 2009; 4:435.

9. World Health Organization. Effects of ionizing radiation. Available at: http://www.unscear.org/docs/reports/2006/07-82087_Report_2006_Web. pdf. Accessed May 5, 2013. 